\title{
HIGH-TEMPERATURE OPTICAL CONSTANTS OF DUST ANALOGUES FOR THE SOLAR NEBULA
}

\author{
S. Zeidler ${ }^{1}$
}

\begin{abstract}
The dust in protoplanetary disks is influenced by many different processes. Among others, heating processes are the most important ones: they change not only the physical and chemical properties of dust particles, but also their emission spectra. In order to compare observed infrared spectra of young stellar systems with laboratory data of hot (up to $655^{\circ} \mathrm{C}$ ) circumstellar dust analogues, we investigate materials, which are important constituents of dust in protoplanetary disks. We calculated the optical constants by means of a simple Lorentzian oscillator fit and applied them to simulations of small-particle emission spectra in order to compare our results with real astronomical spectra of AGB-stars and protoplanetary disks.
\end{abstract}

\section{Background}

Matter in space is subjected to various different temperature conditions. The temperature has a strong effect on the absorption/emission behavior of dust particles (Koike 2006) and this effect could thoroughly help to give explanations of still unidentified bands in stellar spectra (for instance the $13 \mu \mathrm{m}$ band in AGB-star spectra). Band shifts may also indicate the emission originating from different parts of circumstellar disks. Therefore, studying the influence of the temperature on the absorption properties of astrophysical relevant solid materials is an important aim of laboratory astrophysics.

\section{Performing high temperature infrared spectroscopic measurements}

In this project, which is part of the DFG SPP "The first 10 million years of the solar system", we investigated the dependence of the optical constants on the temperature for synthetic bulk materials such as Corundum $\left(\alpha-\mathrm{Al}_{2} \mathrm{O}_{3}\right)$ and Spinel $\left(\mathrm{MgAl}_{2} \mathrm{O}_{4}\right)$ as well as for the natural minerals $\alpha$-Quartz $\left(\mathrm{SiO}_{2}\right.$, from Brazil) and

${ }^{1}$ Astrophysikalisches Institut, Schillergässchen 2-3, 07745 Jena, Germany 


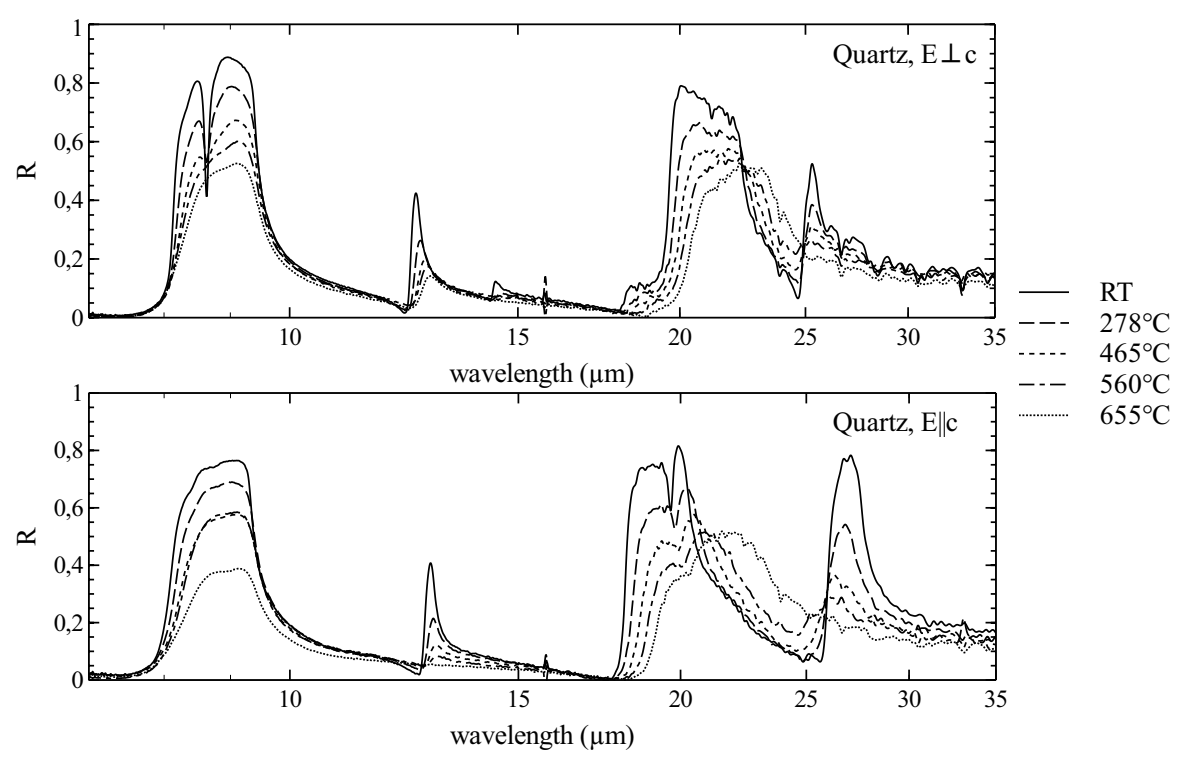

Fig. 1. The measured reflectivity spectra of the natural $\alpha$-quartz crystal in both directions of polarization. At $655^{\circ} \mathrm{C}$ some bands totally disappeared (e.g. the $27 \mu \mathrm{m}$ band in $\mathrm{E} \| \mathrm{c}$ polaritation), which resulted from the second order phase transition to $\beta$-quartz.

Olivine $\left(\mathrm{Mg}_{1.84} \mathrm{Fe}_{0.16} \mathrm{SiO}_{4}\right.$, San Carlos). Corundum and spinel prevail as early condensates in the stellar outflow of AGB-stars and are constituents of the CAIs in meteorites (Fabian 2001). Olivine is a very important constituent of circumstellar dust disks (Juhasz 2010; Henning 2003) around young and evolved stars and of our own planetary system (main constituents of meteorites, Sunshine 2007).

All samples had oriented (except spinel) and polished surfaces to perform infrared reflection-spectroscopy with polarized light. In order to implement these measurements while the samples are heated, we used a special High-TemperatureHigh-Pressure (HTHP) cell, that is built into our Bruker 113v FTIR Spectrometer. The sample chamber of the cell is a cylindric hole in the sample heater of $13 \mathrm{~mm}$ diameter through which the infrared beam can reach the sample. An infrared polarizer has been set right behind the cell in the out-going beam, which performed the reflection measurements under polarized light.

The measurements have been taken at only four different temperatures: room temperature (RT), $278^{\circ} \mathrm{C}, 465^{\circ} \mathrm{C}$, and $655^{\circ} \mathrm{C}$ to prevent the heater from erosion. Only in the case of $\alpha$-quartz we measured also at $560^{\circ} \mathrm{C}$ to get a better temperature resolution of the phase transition to $\beta$-quartz taking place at $575^{\circ} \mathrm{C}$ (see Fig. 1 ).

\section{Derivation of the optical constants}

From the measurements we obtained simple reflection spectra as can be seen in Figure 1. The optical constants (the real part $n$ and the imaginary part $k$ of the 

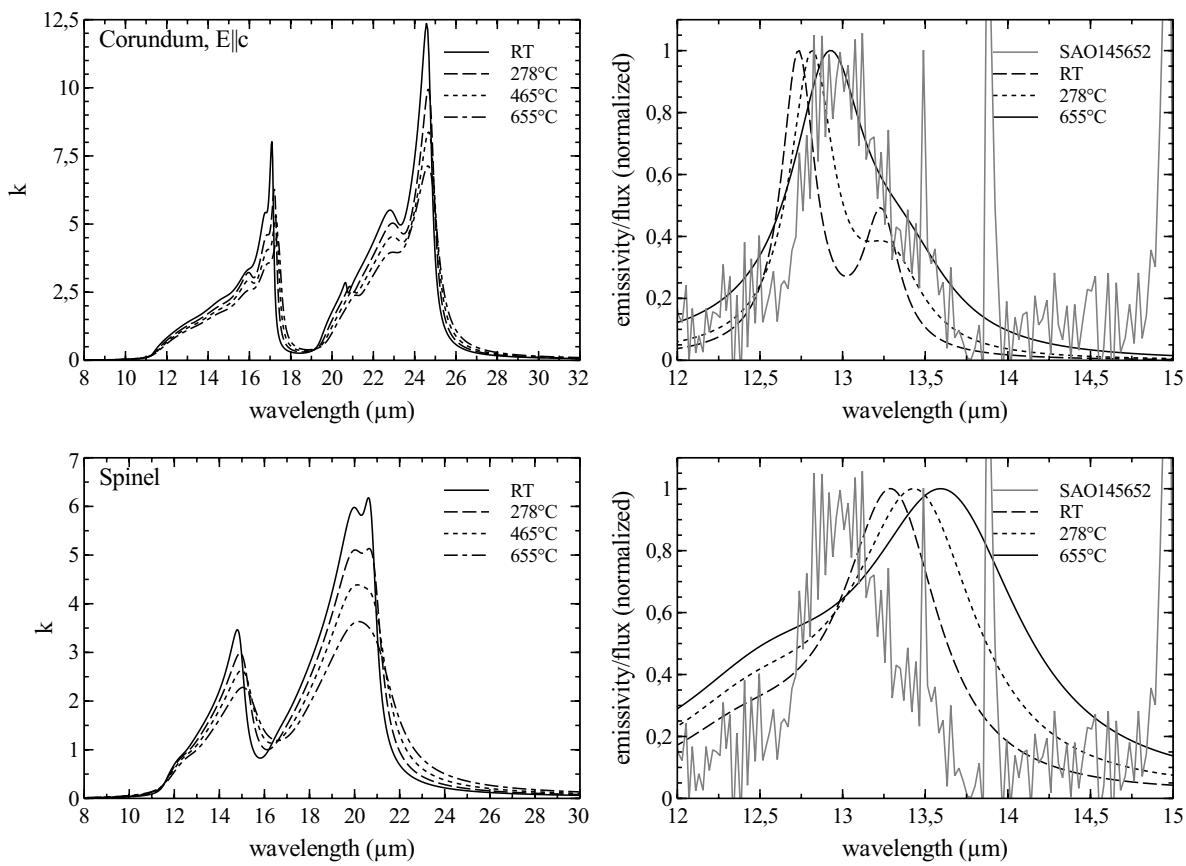

Fig. 2. On the left: imaginary part of the refraction index $N=n+i k$ for Corundum (top) and Spinel (below) from room temperature (RT) up to $655^{\circ} \mathrm{C}$. On the right: comparison between the normalized emission spectra of the $13 \mu \mathrm{m}$ band of the AGB star SAO 145652 and the normalized emissivities of Corundum (top) and Spinel (below) for RT, 278, and $655^{\circ} \mathrm{C}$ (Cabs multiplied by the corresponding Planck-Function).

complex refraction index) have been derived from these spectra with Lorentzian oscillator fits.

Furthermore, the temperature dependence of the fitting parameters itself could be approached by fits of second order polynomials, which resulted in a full analytical description of the temperature dependent optical constants. We also calculated the absorption cross section $C_{a b s}(T)$ for small $(a \ll \lambda)$ spherical particles and multiplied $C_{a b s}(T)$ with the Planck-function at each temperature in order to get the emissivity spectra and to compare the results with astronomical spectra.

The results for Spinel and Corundum, can be seen in Figure 2. The $k$-spectra for Corundum and Spinel show almost the same behavior with increasing temperature. The bands shift to longer wavelengths and the intensity of the bands decreases. They also become slightly broader. We also compared the small particle emissivity spectra of both materials with the emission spectrum of the AGB star SAO 145652, focusing on the $13 \mu \mathrm{m}$ band (see Fig. 2 on the right side). The best reproduction of the $13 \mu \mathrm{m}$ band with respect to the band position and shape is only reached by Corundum at $655^{\circ} \mathrm{C}$, although at this temperature the band of Corundum is slightly too broad to fit the $13 \mu \mathrm{m}$ band of the star perfectly but this effect could 


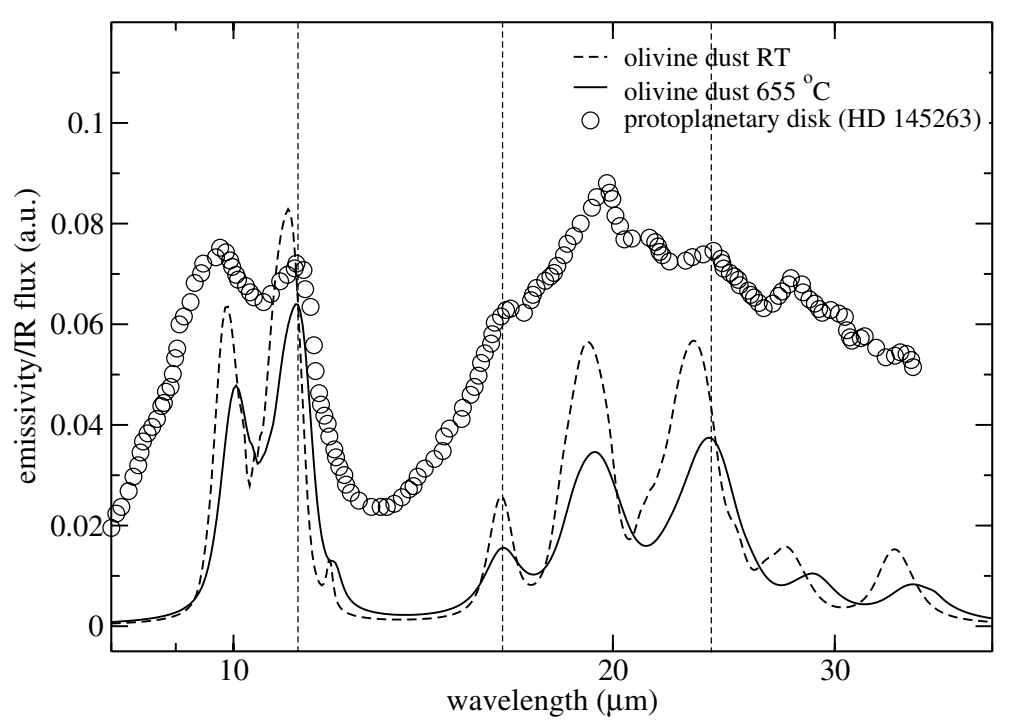

Fig. 3. Comparison of calculated small particle emissivity spectra of olivine at two different temperatures and the emission spectrum of the protoplanetary disk HD 145263.

either be a result of the fittings or has its origin in the noise of the spectrum of the star.

\section{Application to the mineralogy of a protoplanetary disk}

Besides pyroxenes, olivines are the most abundant crystalline minerals in circumstellar dust disks (Juhasz 2010; Henning 2003). However, the exact composition of these olivines is still under discussion (especially the iron content), since the composition itself, the grain shape, and the grain size have a strong influence on the position and the shape of bands. With our investigations we can show, that also the temperature, as an additional parameter, plays an important role for the analysis of spectra of protoplanetary disks and should be taken into account in future discussions about the composition of circumstellar dust.

In Figure 3 we compared the emission spectrum of the protoplanetary disk of HD 145263 (taken with ISO) with calculated small particle emissivity spectra of olivine, based on our measurements at RT and $655^{\circ} \mathrm{C}$. It can be seen that the dust at $655^{\circ} \mathrm{C}$ fits somewhat better the observed emission spectrum than the dust at RT (indicated by the vertical dashed lines, which mark the band positions of olivine dust in the spectrum of HD 145263). Of course we do not claim, that the olivine dust in this protoplanetary disk has a temperature of exactly $655^{\circ} \mathrm{C}$. Beside the other mentioned influences on an emission spectrum, the dust will always hold a certain temperature distribution. But it should be noted, that for the analysis of spectra of circumstellar dust disks, the temperature of the dust should be taken into account just as the shape, size, and the composition of the dust grains. 


\section{References}

Fabian, D., Posch, Th., Mutschke, H., Kerschbaum, F., \& Dorschner J., 2001, A\&A, 373 Juhasz, A., Bouwman, J., Henning, Th., et al., 2010, ApJ, 721, 431

Koike, C., Mutschke, H., Suto, H., et al., 2006, A\&A, 449, 583

Henning, Th. (ed.), 2003, Astromineralogy (= Lecture Notes in Physics, vol. 609) (Springer-Verlag, Berlin and Heidelberg)

Sunshine, J.M., Bus, S.J., Corrigan, C.M., McCoy, T.J., \& Burbine, T.H., 2007, Meteor. Planet. Sci., 42, 155 